\title{
Essais
}

ESSAIS

Revue interdisciplinaire d'Humanités

Hors-série 6 | 2021

Agrobiodiversité et territoires

\section{Cosmopolitiques vitivinicoles, ou pourquoi enlever les guillemets aux vins natures}

Vitivinicultural cosmopolitics, or why take the inverted commas off natural wines

Léo Mariani

\section{OpenEdition}

1 Journals

Édition électronique

URL : https://journals.openedition.org/essais/7803

DOI : $10.4000 /$ essais.7803

ISSN : 2276-0970

Éditeur

École doctorale Montaigne Humanités

Édition imprimée

Date de publication : 1 mars 2021

Pagination : 149-159

ISBN : 978-2-492780-00-4

ISSN : $2417-4211$

Référence électronique

Léo Mariani, «Cosmopolitiques vitivinicoles, ou pourquoi enlever les guillemets aux vins natures », Essais [En ligne], Hors-série 6 | 2021, mis en ligne le 16 mars 2021, consulté le 18 janvier 2023. URL http://journals.openedition.org/essais/7803; DOI : https://doi.org/10.4000/essais.7803 


\section{Cosmopolitiques vitivinicoles, ou pourquoi enlever les guillemets aux vins natures}

\section{Léo Mariani}

Habituellement, on dit d'une personne " qu'elle est nature " lorsqu'elle ne s'embarrasse pas de conventions, quand on veut souligner sa spontanéité et sa simplicité, pas pour sous-entendre qu'elle a grandi dans un environnement naturel, sans humains. Cet emploi de " nature " comme adjectif est aussi fréquent dans le cas des yaourts, où il ne décrit pas non plus la naturalité du produit mais l'absence d'assaisonnement. Contrairement à " naturel ", qui dénote un attribut substantiel, l'adjectif "nature » fait référence à une qualité pragmatique, une façon d'être, sans fard et sans artifice. Dans le cas du vin, où les expressions "vin nature » et "vin naturel » voisinent, l'usage commun semble avoir fait le choix du pragmatisme, préférant l'épithète "nature " à l'évocation d'une hypothétique naturalité. On ne s'étonnera pas, aussi, que la locution " vin naturel » ait quant à elle les faveurs des commentateurs cyniques, à qui elle offre une bonne occasion de rejouer l'un des grands tropismes relativistes : mettre des guillemets à « la nature ». De fait, les guillemets nuancent une idée qu'il faut avoir eue pour la relativiser. Ils introduisent un problème qui n'existe que du point de vue de ceux qui ont à un moment cru en la nature, ou qui croient que d'autres croient en elle, insinuant ici qu'aucun vin n'est vraiment naturel, et qu'il faudrait être au mieux un peu dupe, au pire un peu malhonnête pour penser le contraire.

Les viticulteurs que je connais ne sont pas particulièrement l'un ou l'autre. Ils savent que leurs vins ne sont pas naturels, ils connaissent l'implication que leur production nécessite et ce qu'il en coûte de la négliger. Ils n’ignorent pas, enfin, que les raisins écrasés produisent tout naturellement du vinaigre lorsqu'ils sont laissés sans attention. Bien sûr, cela ne les empêchera pas, à l'occasion, d'affirmer que tel vin est plus naturel que tel autre, lorsque sa production a nécessité moins de chimie par exemple. Mais on ne peut pas leur attribuer la naturalité comme enjeu car ils n'ont jamais cru en la nature. Ils n'ont jamais cherché à être modernes. C'est, pourrait-on dire, le naturel qui leur importe. La naturalité forcément toujours inaccomplie n'en est qu'une implication secondaire. 
Les vins natures ont connu un essor important ces quinze dernières années, mais on ne comprend encore souvent ce succès que comme un engouement dans l'air du temps, une réponse à des préoccupations environnementales de plus en plus pressantes et, partant, comme l'expression d'une quête mythique et de toutes façons illusoire pour une nature qui n'existe pas. Pourtant, si le souci de l'environnement tend à faire produire des vins plus naturels (biologiques par exemple), il ne fait pas nécessairement des vins natures. Qu'on se préoccupe en revanche de rendre le naturel au vin, et toutes mes observations portent à penser que la naturalité comme « le souci de l'environnement » viendront avec. Je voudrais défendre ici l'idée que les vins natures relèvent moins d'une réponse à des injonctions plus ou moins diffuses qu'ils ne posent une question de cosmopolitique ${ }^{1}$, autrement dit qu'ils n'ouvrent une forme de contestation fondamentale, portant sur la définition de ce qu'est ou doit être le vin. C'est donc tout une façon d'être en rapport avec le vivant qui se noue dans ces boissons, et ce rapport fait lui-même dessein, contre le type de rapport imposé par la modernité agricole et ses modes de rationalisation. Au sens le plus général, les vins natures seraient ainsi un symptômes et/ou une réaction à l'emprise que le dessein moderne exerce sur les pratiques et les conceptions du vivant. Ils traduiraient le désir profondément humain de connaitre, et de pouvoir le faire par soi-même. En ce sens, je n'envisage pas le désir au sens platonicien du terme, comme l'épreuve d'une absence ou d'un manque à combler, mais au sens plus " affectif " que lui donnait Gilles Deleuze : un désir producteur et instaurateur, qui tend spontanément à connecter et à libérer des devenirs, mais que des formations sociaux-matérielles viennent ensuite organiser, capter et refréner, introduisant ainsi le manque, et l'impression que le désir en est une conséquence. C'est ce désir fondamental de projection dans le monde qui me paraît s'exprimer dans les vins natures, une intensité affective qui cherche à circuler.

Le naturel n'est donc pas un attribut pensable dans des termes essentialistes. Il n'est pas plus gérable, en pratique, par des modes de viticulture et d'œnologie qui le sont tout autant. Ce n'est pas un contenu que l'on pourrait ajuster ou corriger par une action directe et dirigée, comme on dose les proportions d'un cocktail mais une qualité pragmatique, définie comme un effet qui doit advenir par lui-même ${ }^{2}$. Bien sûr, les vins natures sont faits, parce qu'ils sont préparés, plus précisément suscités, mais il leur revient, par définition, de se déterminer et de se produire à leur façon. Dans les cas qu'il m'a été donné d'observer, c'est leur capacité à le faire qui sera ensuite jugée. Pour qu'ils parviennent à ce point, ils doivent donc être accompagnés, protégés, orientés et préparés.

1 Isabelle Stengers, Une autre science est possible! Manifeste pour un ralentissement des sciences (suivi de Le Poulpe du doctorat), Paris, La Découverte, 2017.

2 François Jullien, Traité de l'efficacité, Paris, Grasset, 1997. 


\section{Natures et divers}

Souvent, on n'attend donc pas d'abord d'un vin nature qu'il soit naturel mais, comme on l'attend d'une personne, "qu'il ne soit pas maquillé "s , qu'il fasse preuve de "spontanéité ", de "sincérité ", qu'il dise ce qu'il est, qu'il soit ce qu'il dit. Et l'on comprend que la chimie pose un problème très différent à ce genre de boisson qu'elle ne le fait à un vin qui se voudrait naturel, ou même simplement biologique. Dans ces derniers cas, le vin est défini par essence, selon sa composition, alors que dans le cas du vin nature il est défini sur un plan phénoménal, du point de vue de ses effets. À chacun, le produit chimique pose donc un dilemme relatif ; problème de nature dans un cas, parce qu'il n'est pas naturel et qu'il remet en cause une définition substantielle ; problème d'usage pour les autres, parce qu'il travestit les apparences et bloque une expression pragmatique. En contribuant à maquiller le vin, le produit chimique bride son expression spontanée et, comme il le ferait avec un visage, il va ainsi à l'encontre de ce qui fait le naturel. Pour preuve de ce déplacement de focale, on peut évoquer l'exemple d'un composé qui, quoique naturel, cause à l'occasion le même type de rejet : l'eau. En période de grande sécheresse, il arrive en effet que des vignerons s'empêchent d'arroser des vignes en état de stress hydrique avancé, simplement parce que cela mènerait à l'artificialisation du vin. Ce n'est pas un choix facile qu'ils réalisent ainsi, de laisser les plantes souffrir et de souffrir avec elles. Ils n'ignorent pas, non plus, que les volumes de production seront impactés, comme leurs revenus d'ailleurs, mais ils ont la conviction que l'addition d'eau viendrait empêcher l'une des relations que la vigne déploie avec son environnement, précisément l'une de ces relations dont un vin "qui ne ment pas » est censé témoigner. La marque d'une sécheresse (petits volumes, concentrations organoleptique et alcoolique plus élevée notamment) contribue à ce qu'on qualifie parfois "d'effet millésime ». Elle caractérise le vin en même temps qu'elle permet d'évaluer la sincérité et l'originalité avec laquelle il traduit l'épreuve.

Pour l'essentiel, le naturel est là, dans cette capacité du vin à faire la preuve (l'expression prend tout son sens ici) de son histoire, à témoigner des relations dont il est, littéralement, le produit en devenir, et dans la possibilité qu'il offre aux humains de pouvoir l'évaluer selon cette ontogenèse. Le manque ou l'excès de soleil, de pluie, la composition des sols, les populations de levures, tous ces liens qui interviennent dans la génération d'un vin sont susceptibles d'y trouver une traduction. Mais là où la viticulture et l'œnologie conventionnelles éliminent et corrigent directement ceux qui lui posent problème pour

3 Ici comme dans la suite du texte, les termes entre guillemets m’ont tous été donnés par des vignerons et des amateurs au cours des quinze années durant lesquelles j'ai réalisé des enquêtes au long cours dans les milieux du vin en parallèle de recherches sur les sensibilités et les rapports à l'environnement en Asie du Sud-Est. 
plaire (aux humains), la viticulture et l'œnologie natures se font un devoir de ne pas toucher l'essence, considérant que l'identité du vin est dans son intégrité et que cette dernière seule garantit qu'il sera capable de se produire, d'advenir d'une façon qui lui appartienne.

Bien sûr, ces objectifs sont ambigus, parce qu'il faut en dernière instance que le vin reste buvable, qu'il ne se transforme pas en vinaigre par exemple ${ }^{4}$. À cette fin, il faudra bien user d'artifices pour préserver certaines de ses qualités, et freiner le développement de certaines autres, par exemple refroidir les moûts pour éviter le développement de levures indésirables. Mais ce sont des approches indirectes, portant sur l'écologie de la boisson et non sur elle-même, qui seront alors toujours privilégiées. Ne pas artificialiser le vin, préserver son intégrité.

Il est amusant que les contempteurs des vins natures se soient acharnés à dénoncer leur manque de régularité, comme s'il était inconcevable que la diversité et la capacité à surprendre puissent être en elles-mêmes sources d'intérêt. Or c'est précisément cette production de singularités qui très souvent importe. Les gens que j'ai interrogés aimaient les vins natures pour leur diversité et leur imprévisibilité, non pas pour les certitudes qu'ils offraient, mais comme un mode spéculatif d'exploration des possibles. Pour les plus radicaux, il fallait à la rigueur que chaque vin puisse être unique, que chacun puisse raconter quelque-chose de différent. C'est le sens du refus de maquillage (la non-addition de soufre notamment) et de la préservation jalouse de l'intégrité du vin, qui exacerbent sa sensibilité, la capacité qu'il a d'être affecté par son environnement et d'exprimer ces (ses) sensations. De ce fait, il n'est pas rare que les bouteilles d'un vin diffèrent les unes des autres, voire qu'une seule de ces bouteilles, une fois ouverte, donne à boire dix nouveaux vins, parce que le liquide se transforme au contact de l'air (il s'oxyde). Le vin nature est un hyper sensible qui ne se cache pas, et les amateurs semblent avoir appris à faire avec cette fragilité (quand ils ne la cherchaient pas), ce pouvoir d'être affecté qui fait la force de témoignage de ces vins, et leur diversité. Ils ont donc accepté d'être parfois déçus, de patienter et de retenter leur chance, quelques minutes ou quelques heures plus tard. Ils ont également accepté de suivre ces vins sur des chemins parfois tortueux ou bizarres, d'autres fois brillants ou éclatants... ce faisant, ils ont revu ce qu'ils pouvaient entendre par «mauvaises surprises», parce qu'ils ont bien voulu repousser les limites de leurs habitudes et de leurs attentes, transformant une première déception en curiosité, un raté en occasion pour un nouvel essai. Les mauvaises surprises impliquent beaucoup que l'on projette quelque-chose de particulier, et tout converge pour que les vins natures n'offrent pas cette possibilité, pour qu'ils

4 Et de fait, beaucoup de vins natures flirtent avec le vinaigre, redéfinissant ce faisant les limites de ce que les dégustateurs sont capables d'accepter. 
ne puissent pas être ceci ou cela, mais toujours potentiels en devenir. À ce titre, ils contribuent à diversifier les formes d'hédonisme : s'ils sont sans doute "mauvais " parfois, ils ne sont pas seulement "bons " le reste du temps mais aussi très souvent "drôles ", " curieux ", " inattendus " ou même "étranges ", non-conventionnels en réalité, et donc difficilement comparables. C'est ce rapport aux normes sur lequel il faut maintenant s'arrêter.

\section{Diversité sans soufre et scalabilité}

Dans son bel ouvrage sur le vin 5 , Georges Guille-Escuret répliquait au Général de Gaulle désespérant de parvenir à gouverner un pays qui, comme la France, produit tant de fromages : n'est-ce pas plutôt parce que la France est ingouvernable qu'elle a pu produire une telle diversité de fromages, se demandait-il en retour ? Les vins (et les vignerons) natures apportent une réponse littérale à ce renversement, une réponse anarchiste (" sans commandement »). C'est que le naturel pose une obligation de fin, non de moyens. Il libéralise les options techniques et, s'il normalise l'ontologie, c'est dans une forme processuelle où les vins sont rendus à des causalités environnementales infiniment plus imprévisibles que celles des humains. La viticulture et l'œnologie moderne, sûres de leurs objectifs, de leurs ripostes et de leurs solutions, normalisent des processus pour produire des vins. Elles solutionnent ainsi les questions que le vigneron peut se poser, et minimisent dans le même temps le pouvoir de leurs vins de les faire hésiter. Ce pouvoir leur est ici restitué dans toute sa plénitude, car c'est le droit de poser ses propres conditions qui est concédé au vin nature par ses partenaires humains consentants : il sera lui-même et pas autre chose. La diversité se nourrit de cette autonomie et de l'exigence que les humains s'adressent à eux-mêmes en se l'imposant. Pour être à la hauteur, ils ne pourront pas s'appuyer sur des recettes qui marchent à chaque fois, des formes de traitement de masse appliquées à des populations homogènes de vignes, de raisins ou de levures. Ils devront concilier avec le particulier, le singulier, faire preuve d'une intelligence adaptative. En se contraignant de la sorte, ils se forcent enfin à produire les conditions de cette autonomie dans un monde agricole normé par des humains. Ce faisant, ils posent les bases de ce qui ne peut exister et se définir que comme un mouvement d'émancipation, des vins et de ceux qui les font. Cette soustraction aux gouvernements techniques, institutionnels et sociaux est inévitable, parce qu'il s'agit fondamentalement de faire passer la force de façonnement et de normalisation de l'environnement devant celle des humains. Ceci explique sûrement que les vins natures soient en général qualifiés par des termes qui

5 Georges Guille-Escuret, La Souche, la cuve et la bouteille. Les rencontres de l'histoire et de la nature dans un aliment: le vin, Paris, Éditions de la MSH, 1989. 
expriment leur caractère individualiste, anti-normatif : " rebelles ", " originaux ", " impolis ", " différents ", " non-conventionnels ", " punks " ${ }^{6}$ ou tout simplement « libres».

Mais on peut s'arrêter, pour illustrer cette idée et la pousser encore un peu, sur la plus fondamentale des libertés conquises par les vins natures, une liberté qui leur vient au moment où l'on décide de leur ôter le soufre. Le soufre est ce composé chimique normalement ajouté lors de la vendange, de la vinification et/ou de la mise en bouteille pour stabiliser les moûts puis le liquide, les rendre moins dépendants des effets de l'oxygène notamment, et les protéger du développement de certaines levures ou moisissures. Le soufre a donc la réputation de protéger le vin (qui en produit d'ailleurs spontanément de lui-même, en petite quantité $)^{7}$, mais on devrait se contenter de dire qu'il le stabilise, car le vin, en soi, n'a pas besoin de protection. Des humains décident, à un moment, de protéger/préserver certaines de ses qualités et de ses potentialités, en même temps qu'ils choisissent d'en refuser/empêcher d'autres. En incorporant du soufre au vin, ils ralentissent son ontogenèse, limitent les rapports qu'il pourra développer avec son environnement et subordonnent sa temporalité à la leur. Le vin est histoire de temporalités. Il a, comme le souligne Guille-Escuret ${ }^{8}$, permis à de grandes dynasties bourgeoises d'ancrer les leurs dans la terre et dans les caves, en les nouant dans un liquide qui prenait de la valeur avec le temps, un stock qui pouvait autrement dit servir la capitalisation de leurs ambitions socio-économiques.

Sans soufre, le vin offre moins de prises à ces logiques, il a d'autres propensions $^{9}$ : devenu plus difficile à conserver ${ }^{10}$, plus instable, plus fragile et moins prévisible, il gagne en présence et en influence, capte plus continûment l'attention et exclut qu'on ne l'oublie au fond d'une cave pour le réévaluer plus tard. C'est lui qui donne le ton désormais, son rythme qui contraint toute l'écologie sensible et technique du rapport, exigeant qu'on lui trouve des débouchés rapides, conciliables avec ses qualités. La vocation de ces vins est intrinsèque-

6 Voir par exemple la mini-série Punkovino récemment diffusée par Arte. Notons, au passage, qu'on a parfois utilisé l'expression de "vins de garages " à propos des vins natures, surtout dans les premiers temps... Probablement parce que c'est souvent dans des garages que les mouvement punks débutent.

7 C'est d'ailleurs souvent un point de réflexion important pour des vignerons qui voudraient «faire nature » mais qui hésitent encore : ils se disent alors que si le vin produit spontanément du soufre, ce n'est pas aller contre sa propension que de lui en ajouter un peu, mais plutôt une façon de l'accompagner. Où l'on voit à nouveau que l'important est de ne pas faire mentir le vin, en essayant de coller au plus près de ses dynamiques propres.

8 Georges Guille-Escuret, La Souche, la cuve et la bouteille, op. cit.

9 François Jullien, Traité de l'efficacité, op. cit.

10 On a évoqué le fait que le vin contraignait également très différemment le travail des vignes et de la cave, qui tendent par exemple à s'adapter au besoin croissant d'acidité (qui améliore la conservation des vins et permet de compenser, un peu, l'absence de soufre). 
ment locale, parce qu'il ne sont pas plus fait pour le transport que pour le stockage et la conservation. Ils sont non-scalables ${ }^{11}$ au sens le plus fondamental du terme, précisément définis selon leur capacité à changer de nature. C'est pourquoi ils forment aujourd'hui un point intéressant pour l'observation de la globalisation en train de se faire, un nœud antagoniste où l'inertie de leurs qualités se confronte à l'aspiration du succès qu'ils rencontrent en France et à travers le monde (au Japon en particulier). On doit se demander, alors, si des vins définis par leur contingence peuvent changer d'échelles et se mettre à valoir pour des gens de plus en plus nombreux sans justement perdre ce qui faisait leur intérêt et leur définition?

L'histoire et l'actualité montrent que cette question du changement d'échelle s'est en général résolue de façon violente, par l'avilissement de populations végétales et humaines à travers le développement de la monoculture et de ses modes de gestion directifs et abstraits ${ }^{12,13,14}$. Or les vins natures ont ceci d'intéressant qu'ils sont, ou ne sont pas. On ne peut les toucher (par exemple leur mettre du soufre pour qu'ils résistent mieux au transport) sans aller contre leur définition même, donc sans leur faire perdre ce pourquoi les gens tiennent à eux. Ils ne sont par conséquent susceptibles d'un changement d'échelle que pour autant que des gens acceptent de faire avec leur caractère contingent, instable et singulier. Peut-être en ce sens que l'accueil très positif qui leur est réservé au Japon en particulier doit être mis en rapport avec un "fond analogique ${ }^{15}$ qui accueille ce genre de qualités avec une bienveillance de principe. Peut-être enfin que cette hypothèse donne à spéculer sur ce à quoi une globalisation plus tolérante à l'impermanence et aux non-humains qui la portent, pourrait ressembler ${ }^{16}$. Mais pour finir, on s'attachera surtout à spécifier un peu certaines des formes d'attention et d'attachement auxquelles les vins natures sont associées, ce pour insister sur la transversalité du biais qu'ils introduisent dans la production des mondes, et qui s'apparente à un réagencement complet de perspectives.

11 Anna L. Tsing, " On Nonscalability: The Living World Is Not Amenable to Precision-Nested Scales ", Common Knowledge, 18(3), 2012, p. 505-523.

12 James C. Scott, Homo Domesticus. Une histoire profonde des premiers États, trad. Marc SaintUpéry, Paris, La Découverte, 2019.

13 Sidney Mintz, Sweetness and Power: The Place of Sugar in Modern History, New York, VikingPenguin, 1985.

14 Anna L. Tsing, "On Nonscalability ", op. cit.

15 Philippe Descola, Par delà nature et culture, Paris, Gallimard, 2005.

16 Je ne suppose pas que cette globalisation serait nécessairement bonne, mais elle serait certainement plus diverse. Comme le souligne Anna Tsing, les relations " transformatives » sont le médium pour l'émergence de la diversité. Que cette diversité soit bonne ou mauvaise est un autre sujet, mais la diversité est bonne en elle-même. 


\section{Sur la piste du naturel. Co-histoire et sensibilités}

Lorsque j’ai commencé à fréquenter les milieux vitivinicoles, une quinzaine d'année en arrière, j’imaginais le vin à la façon essentialiste, comme un produit, donc comme un objet fini qui pourrait être ensuite contemplé, décrit ou représenté de façon plus ou moins habile, plus ou moins culturelle. Cette conception avait sans doute à voir avec les discours dominants, populaires ou mêmes savants, qui les conceptualisent encore souvent dans ces termes. Mais plus encore, je crois qu'elle était très liée aux vins eux-mêmes, ceux auxquels on a le plus communément accès et qui incarnent véritablement ce topos : des vins maquillés, stabilisés pour les besoins de leur commerce et qui se donnent à voir comme des tableaux. Rien d'étonnant, au demeurant, à ce que ces vins dont la temporalité peut coïncider avec celle d'une vie humaine permettent le même genre d'expérience qu'une œuvre d'art quand on les appréhende dans le temps resserré de la dégustation, un instant durant lequel ils resteront égaux à euxmêmes, en suspens, docilement offerts à la contemplation et à la description. Les vins natures proposent une prise plus exacerbée au temps et à ses altérations, donc une accroche moins stable à la perception. Ils ouvrent une ligne de fuite plutôt qu'ils ne délimitent une surface ou un volume : appliquez-vous à les saisir ici, et déjà ils se dérobent là, portés par leur pouvoir d'effectuation et de mise en relation, motricité inhérente qui invite l'attention sur d'autres pistes, suggérant d'autres formes de rapports. L'amateur de vins natures, comme le vigneron, est souvent "à la manière du chasseur-cueilleur ", il fait avec les choses qu'il laisse se faire, et s'oriente «le long d'une ligne [qu'il suit] plutôt que sur un plan [qu'il embrasserait du regard] (Leroi-Gourhan cité par Ingold ${ }^{17}$ ).

Ces rapports ne sont pas simplement donnés par les qualités organoleptiques du vin. Ils dépendent aussi, et comme on l'a dit plus haut, de la possibilité d'ancrer ces qualités dans une histoire relationnelle dont le vin est censé témoigner. Dans les premiers temps de mes enquêtes, je me demandais pourquoi les vignerons dédaignaient toujours très ostensiblement les dégustations à l'aveugle, ces tests qui servent à l'évaluation des vins. On répondait à cette question en évoquant des blessures d'ego, ou la difficulté bien compréhensible d'être mis en compétition, comparé. Si cette interprétation n'est sûrement pas tout à fait déplacée, elle reste trop superficielle car, à bien y regarder, ce sont en fait les modalités même de l'évaluation qui posent problème.

Les dégustations à l'aveugle instaurent une relation de face-à-face entre le vin et le goûteur. Elles créent des conditions très particulières (très scientifiques) qui visent à neutraliser tous les éléments de contexte, toutes les formes de causalités environnementales qui pourraient interférer sur le jugement. Ce faisant, elles ne se contentent pas de créer les conditions pour évaluer des vins et mesurer des goûts, elles définissent ce que les uns et les autres sont : une essence pour les premiers, la représentation d'une sensation déduite de cette 
essence pour les seconds. Ces goûts et ces vins abstraits ne signifient pas grand chose pour des vignerons avec lesquels j'ai travaillé, parce que leurs modes d'objectivation consistent à faire exactement le contraire, en inscrivant le vin dans les relations que le dispositif de la dégustation à l'aveugle neutralise ${ }^{18}$. Alors, le vin n'a d'intérêt que dans le rapport différentiel qui s'établit avec l'ensemble de ses conditions de production, celles de l'instant où il s'effectue, et celles qui l'ont porté jusque-là : les conditions de son ontogenèse, de la co-histoire dont il doit témoigner. Plus qu'un jeu d'abstraction de qualités sensibles, il faut concrétiser le vin, lui donner des coordonnées. Lui-même est censé participer à cette épreuve du naturel, et c'est pourquoi il ne faut pas que ses failles aient été dissimulées, maquillées. Au contraire, ce sont elles qui lui donnent sens et beauté, d'une façon comparable à celle qui se fait jour lorsqu'on apprend à comprendre/connaître une personne, en partageant des moments avec elle, en s'informant de son passé et en donnant ainsi des références aux apparences physiques et morales (cicatrices, rides, comportements, etc.) que le temps a forgé et qu'aucun maquillage ne vient dissimuler. Souvent, les vins natures sont plus attachants voire émouvants que "beaux ", parce qu'ils ne sont pas parfaits, qu'ils n'essaient pas de cacher le fait qu'ils ne le sont pas, et que ce n'est pas ce qu'ils visent.

\section{La diversité contre l'État?}

Les vins natures ne sont pas forcément le fruit « d'un souci pour l'environnement ". Souvent, ils me semblent faire écho à quelque-chose de plus fondamental et de plus sensible à la fois : le besoin de faire relation, d'explorer et de texturer des rapports. Le "souci » ne renvoie donc pas à "l'anxiété » ici, mais à "l'être concerné" ( "matter of concern $\left.{ }^{19}\right)$, ce qui prête à un jeu de mot qui n'est pas innocent : être concerné, et concerner l'être lui-même : se donner d'autres existants comme partenaires intègres et autonomes. Envisagés en ce sens, et replacés dans le champs des possibles, par rapport aux autres potentialités de "faire vin ", les vins natures sont un échappatoire ou un plaidoyer cosmopolitique qui libère des façons alternatives de définir et de connaître, non seulement le vin mais aussi l'environnement, le goût et les pratiques associées. Beaucoup de ceux qui font ces vins ne veulent pas appliquer ou respecter d'autres normes que celles qui sont produites par leur environnement lui-même, et ils ne se satisfont plus du triptyque scientifique-technicien-agriculteur mis en place par la modernité agricole pour cadrer la production. On peut dire qu'ils revendiquent le droit anarchiste et libéral d'être leurs propres décideurs et, ce faisant, celui d'être chercheurs : de ne pas seulement produire mais d'explorer.

18 À ce propos, voir aussi : Geneviève Teil, "Quand les acteurs se mêlent d'ontologie ", Revue d'Anthropologie des Connaissances, 5(2), 2011, p. 437-462.

19 Bruno Latour, "Why Has Critique Run out of Steam? FromMatters of Fact to Matters of Concern », Critical Inquiry, 30, 2004, p. 225-248. 
On entend beaucoup qu'il est temps d'inventer d'autres récits sur le monde. L'exemple des vins natures, qui est un exemple de réouverture des possibles, suggère que cet objectif est plus facile à atteindre en se donnant aussi, et peutêtre avant tout, d'autres partenaires, en rendant aux choses du monde «le pouvoir de nous faire hésiter ", et même un peu plus ici : le pouvoir de nous imposer leurs conditions. Que l'on décrète qu'il faut se soucier de l'environnement, prendre soin de la terre, et l'on devra travailler à incarner cette abstraction dans des réalisations qui auront pour principal moteur des motivations humaines qu'il faudra sans cesse renouveler, ressusciter, réalimenter, comme dans un jeu binaire à somme nulle : abstraction, application, abstraction, application. L'exemple des vins natures esquisse une piste alternative, parce que le souci n'y est pas une cause mais une conséquence : on se le fait advenir en prenant comme ressort la motricité intrinsèque du vivant autonome. Autonome ne veut pas dire abandonné à lui-même, bien sûr, mais contrôlé différemment, en mobilisant d'autres formes de causalités et d'efficacité (qui sont il est vrai plus coûteuses en temps et en énergie mais dans le même temps plus intéressantes, i.e. plus susceptibles d'intéresser au destin de la terre), des modalités qui accompagnent et qui entourent. Ainsi n'est-ce sûrement pas un hasard si les formes d'agrobiodiversité les plus développées semblent toujours liées à ce genre de configurations où la relation entre les humains et les plantes s'appuie sur des modes d'action indirects et donc respectueux de l'intégrité des végétaux plutôt que sur des modes directs ${ }^{20}$ adressés à l'essence. La lecture du dernier ouvrage de James $S \operatorname{cott}{ }^{21}$ pourrait également convaincre que ces formes se déploient en général " contre l'État » contrôleur et administrateur, avec lequel elles sont dans une sorte d'incompatibilité fondamentale.

Léo Mariani

Muséum National d'Histoire Naturelle Laboratoire d'Éco-anthropologie (UMR 7206)

leo.mariani@mnhn.fr

Léo Mariani est anthropologue, enseignant-chercheur au Museum National d'Histoire Naturelle. Ses recherches se situent à la croisée d'une anthropologie du corps, de l'environnement et de l'alimentation, et sont influencées par la philosophie et l'histoire. Il a longtemps résidé et enquêté dans plusieurs pays d'Asie du Sud-Est, notamment sur un fruit à la mauvaise réputation olfactive (le durian). Parallèlement à ces recherches, il a travaillé dans les milieux vitivinicoles du Sud de la France (comme ouvrier agricole et comme commercial en France et à l'international), où il a réalisé des enquêtes. Il est l'auteur de plusieurs articles, d'un ouvrage d'anthropologie et d'histoire politique, et il a codirigé, avec Carine Plancke, un volume sur les affects.

20 André-Geroges Haudricourt, « Domestication des animaux, culture des plantes et traitement d'autrui ", l'Homme, 2(1), 1962, p. 40-50.

21 James C. Scott, Homo Domesticus, op. cit. 


\section{Résumé}

Les vins natures ont connu un essor important ces quinze dernières années. On ne comprend souvent ce succès que comme un engouement dans l'air du temps, une réponse à des préoccupations environnementales de plus en plus pressantes et, partant, comme l'expression d'une quête mythique et de toutes façons illusoire pour une nature qui n'existe pas. Cet article-essai propose plutôt de les considérer comme un mode pratique et spéculatif d'exploration des possibles, une façon originale de faire relation qui interroge les goûts autant que les normes de gouvernance.

\section{Mots-clés}

Vin, nature, naturel, ontologie, diversité, matérialisme.

\section{Abstract}

Nature wine making has been on the rise over these past fifteen years, but its success is often interpreted as a fad, a reaction to environmental concerns, and, consequently, the expression of a fantasy quest for a nature that does not exist. In this article, I claim that nature wines should rather be considered as the expression of a cosmopolitical plea, a pratical and speculative mode of exploring possibles.

\section{Keywords}

Wine, Nature, Natural, Ontology, Diversity, Materialism. 